\title{
LegalTech in a Law Office in the context of Standardization and Autonomic Intelligence
}

Michat Wódczak

\section{Introduction}

In the light of the discussions accompanying the undoubted multitude of aspects behind the concept of LegalTech analyzed from a futuristic perspective, basically covering not only the operation of a law firm office per se, but, in a holistic sense, also broadly understood processes, such as the creation and application of law, or even the interpretation thereof, one may consider certain analogies with the works ongoing over many years on convergence and automation of telecommunications systems, where the concept of autonomic intelligence ${ }^{1}$ is introduced under the umbrella of the Future Internet, going even beyond what is expected from artificial intelligence. Due to an extensive nature of this phenomenon understood in such a way, it seems necessary to explore this issue in terms of standardization $^{2}$, which, especially in a historical understanding, may provide all the desirable directions necessary for a proper placement of not only the legal aspects, but also the related technological factors.

\section{Legal Services and Standardization}

According to factual circumstances, with the passage of time one could discern a conspicuous alteration in the business model ${ }^{3}$ applicable to the operation of modern law offices, which is related to the more and more

1 The concepts contained in this work, apart from references to specific citations, have been outlined on the basis of the monograph Michał Wódczak, Autonomic Intelligence Evolved Cooperative Networking (Wiley 2018), as well as the lectures carried out by its author under the umbrella of the Samsung-SGH Business Course organized in cooperation with Warsaw School of Economics.

2 Richard Susskind, Tomorrow's Lawyers. An Introduction to Your Future (Oxford University Press 2nd edn, 2017) 134.

3 Tanel Kerikmäe and others, 'Legal Technology for Law Firms: Determining Roadmaps for Innovation' (2018) Croatian International Relations Review 105. 
advanced processes of task automation that so far have seemed to be typically a human domain, just to mention the entire spectrum of solutions related to the exchange of electronic documents. As a consequence, such a conversion seems to naturally translate into a new approach to the model of legal service provisioning 4 , to be organized not exactly literally "in the law office", but rather "by the law office", allowing to offer a better value for a lower price, and, at the same time, to obtain the so much desirable competitive advantage ${ }^{5}$. However, such an approach is connected with specific challenges, since two, somewhat interweaving areas, are subject to a mutual change, both the one pertaining to legal business, and the one related to the technological operation of the same.

Given such a context, in principle, it appears fairly appropriate to adopt the assumption that computer systems dedicated to law offices exercising the LegalTech orientated approach should not display any closed nature, in the sense of limiting their operation to the area of one country only, but on the contrary, following the example of modern telecommunications systems, just to mention the $5 \mathrm{G}$ technology, should enable, in compliance with all the cybersecurity rules, cooperation on a cross-border, if not a global basis. Such a goal may be achievable by means of a standardization ${ }^{6}$ carried out in a proper manner, as the omission or disruption thereof could have far-reaching consequences. Yet, in the case of such endeavors, there may be no shortcuts, the prove of which may be derived from the fact that the telecommunications systems adopted as the point of reference, where the autonomic intelligence is supposed to be applied, are still at the research and standardization stage.

Therefore, narrowing the scope of consideration down to the most appropriate LegalTech 3.0 stage, as well as taking into account the level of expansion and distribution of the aforementioned computer systems, which are supposed to support the work of a lawyer in the already highlighted aspects of their activity, it appears advisable to place special emphasis on the extremely important role of organization of the said standardization process, since today's level of advancement of telecommunications systems

4 Qian Hongdao and others, 'Legal Technologies in Action: The Future of the Legal Market in Light of Disruptive Innovations' (2019) Sustainability 9.

5 Deloitte Legal, 'What's your problem? Legal Technology' (2018) Legal Management Consulting 4.

6 From now on, unless a technological understanding has been clearly indicated, standardisation shall be perceived as a dual process, pertaining to the area of LegalTech, encompassing both the realm of legal activity and the technological solutions supporting such an activity. 
is a derivative of lessons from not too distant history, when in the 1980s it became conspicuous that globalization requires universal solutions. As a result, a Reference Model for Open Systems Interconnection ${ }^{7}$ was born, the degree of complexity of which, as well as the fluctuations at its research stage, resulted in the fact that standards were not developed at the right time, which is best illustrated by the concept of the so-called apocalypse of two elephants ${ }^{8}$ (Fig. 1).

Despite the relatively expressive ${ }^{9}$ name, the concept of the apocalypse of two elephants is, in fact, intended to illustrate the mutual relationship among three phases, with an emphasis on the standardization stage being located in the middle, while, at the same time, remaining in relation to the research and investment stages, allowing the avoidance of two significant risks. On the one hand, too early standardization, i.e., before the research works have been completed, would undoubtedly lead to potential solutions that could take into account only some of the demands of respective participants of such a process, which could result in attempts at introduction of incompatible products. On the other hand, too late standardization, i.e., overlapping at least partially with the investment stage, would similarly result in incompatible products that would fail to address the demands of all interested parties alike, although this time it would result from a lack of timely arrangements.

7 ITU-T, 'Series X: Data Networks and Open System Communications. OSI Networking and System Aspects - Efficiency' (1998) ITU-T Recommendation X.630 10.

8 Andrew S Tanenbaum and David J Wetherall, Computer Networks (Prentice Hall 2011) 51.

9 Due a specific nature thereof, the original naming from Andrew S Tanenbaum and David J Wetherall, Computer Networks has been maintained, while possibly, from a semantic perspective, the "collision of two elephants" could be a more adequate term. 
Fig. 1. Apocalypse of two elephants

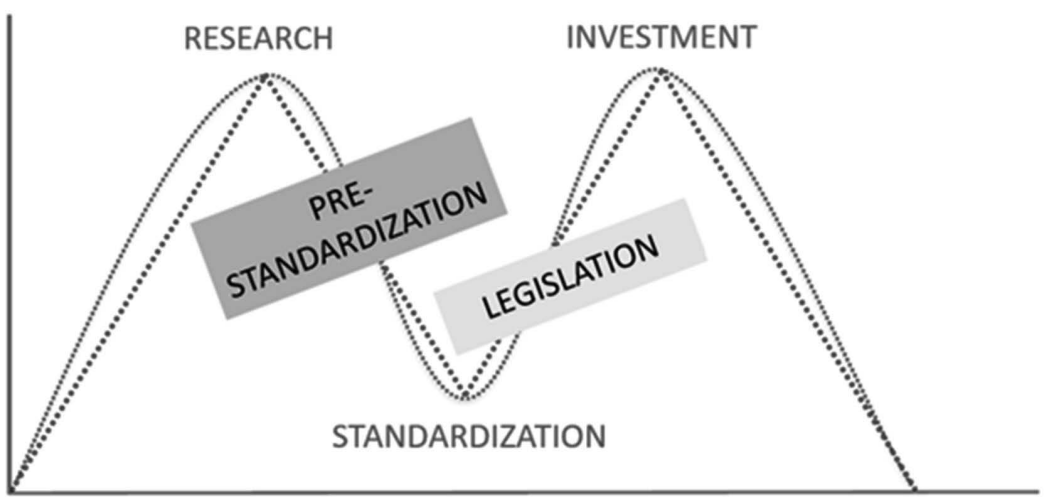

Source: Own elaboration ${ }^{10}$ based on Andrew S Tanenbaum and David J Wetherall, Computer Networks, (Prentice Hall 2011) 52.

At the same time, one should note that this model, also in relation to LegalTech, is not intended to deprive the above-mentioned product recipients of the possibility of using the advantages and benefits of competition, yet solely to ensure its cost-optimal nature. In other words, the currently prevailing trend on the market of telecommunications devices, manufactured for mobile operators, follows the assumption that devices coming from any vendor should work together without any difficulties. Therefore, standardization refers to the interfaces between functional blocks ${ }^{11}$, typically referred to as black boxes, whose operating principles are often protected by patents, at the same time providing a field for obtaining the aforementioned competitive advantage, resulting, for example, from the use of more advanced algorithms. One should expect that such a model shall be assumed for the specifications created for autonomic distributed systems dedicated to LegalTech.

10 The source version does not contain the pre-standardisation and legislation stages.

11 Michał Wódczak and others, 'Standardizing a Reference Model and Autonomic Network Architectures for the Self-Managing Future Internet'(2011) 25(6) IEEE Network 51. 


\section{Technology and Legislation}

Despite emphasizing above that standardization, as understood from the perspective of the functioning of a law office and embedded in a broader context of the entire legal ecosystem, is a dual process, i.e. it should be approached both from the point of view of legal services and technological solutions, one shall realize that the general assumptions regarding such a process remain invariable. A possibly good example could be constituted by a legal service pertaining to the preparation of a contract of a relatively common nature, where the law office could apply an approach based on a template 12 , which would certainly reduce the unit cost in relation to a completely "bespoke" document, although equivalent in terms of content. However, only the circulation of such documents in electronic form, for example, for the purpose of the cooperation between or among two or more law offices, in the case of handling large-scale cases, could reveal the true essence of standardization.

In other words, by a complete analogy to the technological viewpoint, which is undoubtedly a derivative of the interactive or rather transactional model of cooperation, created in this way by a network of law offices, one comes to a situation in which entities, be it legal or technical, exchange information in a known format that is a direct implication for the existence of standardized "interfaces" between or among related lawyers or devices. Given such an approach, a lawyer of a given law office, being subject to exploiting specific experiences or established practices, yet different from equivalent "resources" applied by lawyers of other law offices, shall be perceived as performing their part of the overall undertaking in a way that reflects or imitates the functioning of networked devices ${ }^{13}$, which often may be operating in accordance with proprietary algorithms, so that, on a certain level of abstraction, it shall be possible to offer both services and functionalities adequate to expectations.

In general, the approach outlined above has not been successful from the very outset, and, in reality, it could only become implemented over the years, as exemplified by the aforementioned Reference Model for Open Systems Interconnection, in the case of which one may only recently conclude that present telecommunications systems are becoming the actual incarnation thereof. Paradoxically, the assumptions made several decades ago could be finally fulfilled, which may be a kind of warning for LegalTech

12 Susskind, (n 2) 28.

13 Michał Wódczak, Autonomic Cooperative Networking (Springer 2012) 62. 
solutions, although the acquired knowledge allows the introduction of the pre-standardization stage, whose role is to soften the connection between the first two ones (Fig. 2). Unfortunately, modern telecommunications systems pose new challenges related to cybersecurity on an unprecedented scale, which is attempted to being addressed through appropriate legislative work at the European Union ${ }^{14}$ level, as well as separate legislative processes of the Member States.

\section{Fig. 2. Role of pre-standardization}

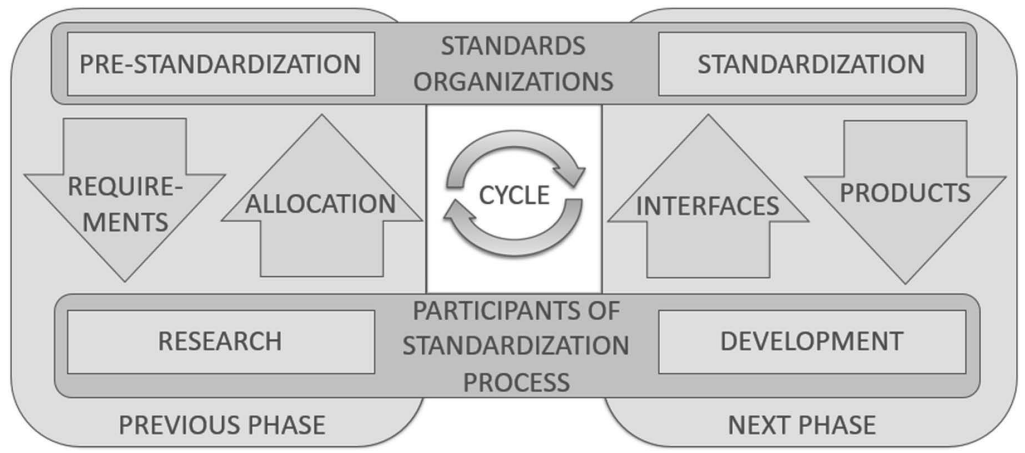

Source: Own elaboration ${ }^{15}$ initially presented under the umbrella of Samsung-SGH Business Course

The context introduced in this way is intended to emphasize the fact that the concepts of the future developed for the needs of the area of LegalTech will be able, given the technical side, to capitalize on the already complete standardization achievements developed for the needs of telecommunications systems, as well as to emphasize the challenges not only in the area of standardization, related to specific technical requirements, but most of all, to stress the legislative demands, which, moving with the times, shall also become reflected in the aforementioned concept of the apocalypse

14 Regulation (EU) 2019/881 of the European Parliament and of the Council of 17 April 2019 on ENISA (the European Union Agency for Cybersecurity) and on information and communications technology cybersecurity certification and repealing Regulation (EU) No 526/20133 [2019] OJ L151 (Cybersecurity Act).

15 In the case of presented approach one may realise that due to their advancement at the standardisation stage more development than typically research works shall be referred to. 
of two elephants. While the exact location of the legislative stage could require a broader discussion, and it will certainly become clear in a longer term, it is already possible to point out, without hesitation, that this stage shall precede the investment stage and, at the same time, overlap with the standardization one, creating somewhat a mirror image of the pre-standardization phase, as shown in Fig. 1.

\section{Legal Processes and Autonomics}

Moving to the aspects related to the potential application of the principles governing the concept of the autonomic intelligence mentioned at the beginning to LegalTech systems, as well as to show the potential of this approach in synergy with solutions based on artificial intelligence, it appears necessary to properly understand the notion of autonomics ${ }^{16}$ in the first place. A fairly common way of defining such an autonomic system is to indicate and underline the possibility thereof to function without any need for an external support, which, as it will become conspicuous soon, may not be an unambiguous definition at all, however, any inaccuracies of the same may be relatively easily explained on the basis of workings of the English language, where there are several similar concepts characterized by similar semantic fields. In fact, those are the slight semantic differences to be responsible for making the resulting ambiguities result in the misunderstanding of the idea of autonomics.

Therefore, the aforementioned autonomic system, also implemented for the purposes of automating legal processes, shall be perceived as imitating the functioning of the biologically-rooted autonomic nervous system in a form similar to what may be found in the human body, although under the assumption of a significantly lower level of complexity thereof, at least in relation to what is attainable by the technology of today. Fairly frequently one may also come across a more elevated form of introducing the definition of autonomics, where it becomes visualized by analogy to the "behavior" of an ant colony ${ }^{17}$. However, an autonomic system should not be confused with either an autonomous system or an automated system, because, in the first case, it is usually referred to as a part of a larger system

16 Michał Wódczak, Autonomic Computing Enabled Cooperative Networked Design (Springer 2014) 3-4.

17 Jeffrey O. Kephart and David M Chess, 'The Vision of Autonomic Computing' (2003) 36(1) IEEE Computer 44. 
that can function "independently", so in the sense of being "detached", while, in the second case, it is thought of more as a system based on the processing of computer scripts.

As a result of said ambiguities, somewhat a synonym for the concept of autonomics in the form of self-management was also introduced, which immediately highlights the difference between the above-mentioned types of systems. Moreover, bearing in mind the earlier reference to possible synergy with artificial intelligence, it is also worth noting, and this will be additionally confirmed by the architectural assumptions adequate for LegalTech systems as mentioned below, that even the concept of autonomic self-learning ${ }^{18}$ systems, referred to in legal literature, also seems not to entirely exhaust the assumptions behind autonomics in the very sense in which it has been introduced in this work. These assumptions are profoundly rooted in the Generic Autonomic Network Architecture ${ }^{19}$ based on the so-called mechanism of Hierarchical Control Loops (Fig. 2), which will be also applicable to the discussion of the concept of autonomics in relation to the functioning of a law office.

In general, the architecture under discussion is based on the assumption that there are four levels of said Hierarchical Control Loops ${ }^{20}$ located at the protocol level, function level, node level, and network level. In each case, remaining in line with the generalized concept of such a Hierarchical Control Loop as outlined in Fig. 3, the superior or controlling role is attributed to and performed by the so-called Decision Elements. As such, Decision Elements can enter into two types of mutual relations in the sense of becoming dependent either vertically or horizontally, which, in the first case, shall be perceived as a relationship of being superior or subordinate, while, in the second case, shall translate into a relationship of a mutual dependence or, rather, being concurrent. It is worth noting that, as far as the technical aspects are concerned, each level is standardized in

18 Expert Group on Liability and New Technologies - New Technologies Formation, 'Liability for Artificial Intelligence and Other Emerging Digital Technologies' (2019) European Commission 25.

19 ETSI-GS-AFI-001, 'Autonomic network engineering for the self-managing Future Internet (AFI); ScenariosUse Cases and Requirements for Autonomic/Self-Managing Future Internet' (ETSI Group Specification 2011) 6.

20 ETSI-GS-AFI-002, 'Autonomic network engineering for the self-managing Future Internet (AFI); Generic Autonomic Network Architecture (An Architectural Reference Model for Autonomic Networking, Cognitive Networking and Self-Management)' (ETSI Group Specification 2011) 13. 
detail, which may facilitate its transposition to a computer system intended to support a pertinent network of law offices.

\section{Agent Systems and Definition of a Thing}

However, in the case under discussion, apart from the technical dimension, largely boiling down to a communication system, there remains the already highlighted strictly legal aspect, which requires some kind of a mapping of the principles of functioning of a future law office in a broader context, where at least some of the legal competences can be expected to be replaced by robotic systems. It seems that due to the fact that the logical structure of the network of such law offices would coincide with the hardware infrastructure of the related computer system, the models of autonomic control of each of them would not so much function in parallel, but could even interweave on the basis of synergy, yet a correct design of such a of such a system would require to carry out, in the first place, the research stage, and only after the appropriate critical mass has been achieved, to commence the standardization path, preferably taking into account pre-standardization. 
Fig. 3. Autonomic Hierarchical Control Loop

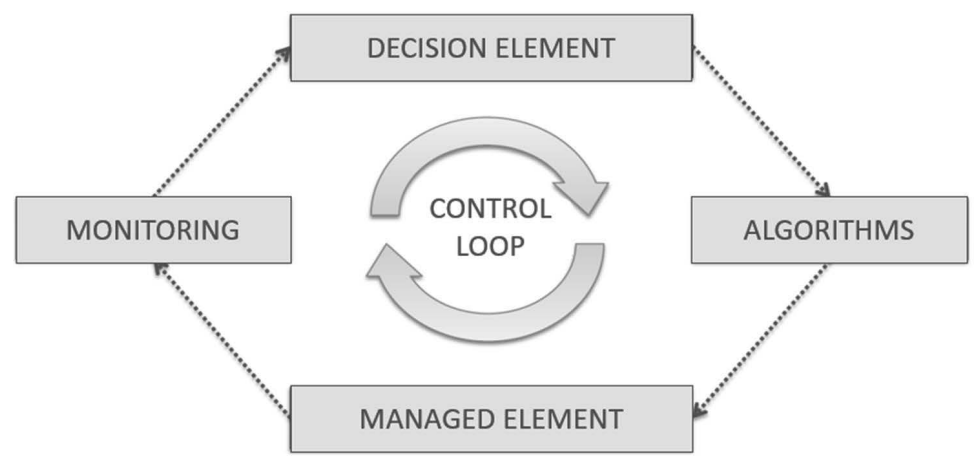

Source: Own elaboration ${ }^{21}$ based on ETSI-GS-AFI-002, 'Autonomic network engineering for the self-managing Future Internet (AFI); Generic Autonomic Network Architecture (An Architectural Reference Model for Autonomic Networking, Cognitive Networking and Self-Management) (2013) ETSI Group Specification 44.

The intelligence of such an autonomic system would consist precisely in the assumption that its individual Decision Elements would have a significant freedom of action and could theoretically not be able to stop certain "behaviors" without intervention, especially should appropriate principles of operation be not assigned authoritatively. The easiest way to explain this phenomenon would be to refer to the operation of the human organism, or more specifically its autonomic nervous system, in the case of which, for example, a stressful situation would cause an accelerated heart rate, which, unfortunately, could not be remedied only by the act of thinking, i.e., by using the brain, but which could spontaneously disappear in an unknowing manner. The aforementioned ant colony would also function similarly, so that encountering an obstacle while moving, it would not

21 The presented high level view of the Hierarchical Autonomic Control Loop shall be perceived as a significantly simplified version thereof, prepared in order to outline its generalised workings. Such an approach is advantageous because it allows to highlight the difference between autonomic intelligence and artificial intelligence, where the latter would be limited to residing within one or more Decision Elements, while the former is based on the interaction of an entire arrangement of such Hierarchical Autonomic Control Loops. 
stop locally, but continue as a consistent whole, remaining in an amazing symbiotic relationship with the environment.

Most obviously, one may consider certain "safety valves" that would allow the administrator or operator, who in principle should only supervise such a system without interfering with its workings, to perform the operation of opening 22 a Hierarchical Control Loop to perform any required adjustments, should there arise a need for doing so. In this context, an important issue from the legal point of view could be related to a certain kind of functional correspondence between the autonomic system using Decision Elements and an agent system ${ }^{23}$. This is so as in the case of a telecommunications operator's network, at the current stage of technology, one refers almost exclusively to completely virtual entities, i.e., occurring only in the form of software, while considering the application of the introduced architectural concepts directly to the area of LegalTech, one could possibly imagine the use of robots, which could raise reasonable questions about their actual legal status.

The emergence of such a question should be interpreted as a harbinger of the aforementioned need to introduce the concept of legislation into the discussed process of standardization of technological systems, while maintaining previous references to the stages of research and investment. After a more in-depth analysis, it may transpire that depending on the features attributed to the agent in question, it can consequently be considered not only as an object, but also as a subject of a legal relationship, and thus qualify, in a large generalization, either as an artificial agent or a moral agent, where, in the first case, the imitation of human intelligence may seem to be sufficient only to exhaust the definition of a thing within the meaning of Art. 45 of the Polish Civil Code, for example, while in the second case, the basis for classification is the recognition of the autonomic flavor of the agent which is related to the fact that it is not dependent on human decisions.

22 ETSI, 'Generic Framework for Multi-Domain Federated ETSI GANA Knowledge Planes (KPs) for End-to-End Autonomic (Closed-Loop) Security Management \& Control for $5 G$ Slices, Networks/Services' (2020) 6 White Paper 12.

23 Frances MT Brazier and others, 'Agents and Service-Oriented Computing for Autonomic Computing: A Research Agenda' (2009) 13(3) IEEE Internet Computing 83. 


\section{Conclusion}

All in all, despite the existence of an evident, although somewhat futurological, mutual correspondence between the network of law offices and the network of computer entities, it is supported by in the strict sense, one should not forget about technical aspects mostly attributable to the legal side, such as for example datafication, algorithmic solutions, or distributed ledgers which should undoubtedly be integrated into the technical part, too. In a sense, one may be under an impression that the degree of conceptual complication, not to mention any possible deployment, may be surprisingly high in the case of the synergistic approach under consideration. Nevertheless, it also transpires that there is no escape from automation in one form or another, and success can only be brought about by a conscious deployment of a well-prepared action plan with a standardizing tone, constructively drawing on the experience of previous years as it has been already indicated. 